\title{
Arvenses asociadas al cultivo de tomate (Solanum lycopersicum L.) en el distrito de Los Santos, República de Panamá
}

\author{
Weeds associated with the tomato crop (Solanum lycopersicum L.) in the district of \\ Los Santos, Republic of Panama
}

Orlando O. Osorio - Burgos ${ }^{1}$, Maira E. Díaz-Vergara ${ }^{2}$

\begin{abstract}
RESUMEN
Las arvenses asociadas al cultivo de tomate (Solanum lycopersicum L.) representan una amenaza significativa para los sistemas de producción agropecuarios y la importancia de su manejo, basándose en el nivel de daño económico, está fuertemente sustentado en la agricultura a nivel mundial.

El objetivo de este trabajo fue la identificación de las arvenses, asociadas al cultivo de tomate, dada su relevancia en los agroecosistemas del Arco Seco de Panamá. Se colectaron muestras en 10 fincas, ubicadas en Tres Quebradas, Las Cruces, La Colorada, El Guásimo, Villa Lourdes, La Espigadilla, Los Ángeles y Santa Ana, ubicados en el Distrito de Los Santos, Provincia de Los Santos (Panama). Del 100\% de las muestras de arvenses asociadas al cultivo de tomate, colectadas en el Distrito de Los Santos, el 17\% corresponde a la familias Euphorbiaceae; 22\% Asteraceae y Fabaceae, (11\% c/u); $8 \%$ Poaceae; $18 \%$ Convolvulaceae, Malvaceae, Solanaceae (6\% c/u ); $20 \%$ Amaranthaceae, Boraginaceae, Commelinaceae, Cucurbitaceae, Sterculiaceae ( $4 \%$ c/u); y el 15\% restante se encuentra distribuido homogéneamente entre las familias: Bignoniaceae, Capparidaceae, Cyperaceae, Loganiaceae, Onagraceae, Plantaginaceae, Portulacaceae, Rubiaceae Scrophulariaceae, Tiliaceae, Verbenaceae, Violaceae, Zygophyllaceae, Las arvenses identificadas pueden actuar como reservorio de entes biológicos, que debe ser consideradas en el diseño de estrategias de manejo agronómico del cultivo.
\end{abstract}

Palabras claves: arvenses, identificación, familias botánicas, especies.

\begin{abstract}
Weeds associated with the tomato productions in the country of Panama represent a significant threat to agricultural activities and the importance of its management related to this crop, based on the level of economic damage is strongly supported in agriculture worldwide.

The main purpose of this article is the identification of different varieties of weeds associated to the tomato crop, given its relevance in the agroecosystems of the Dry Arch zone (a very dry area located in the mid provinces of the republic of Panama which are: Herrera, Los Santos, some parts of Coclé and some parts of Veraguas). Where several samples of different varieties of weeds were collected in ten (10) different tomato fields, such areas are located in different parts of Los Santos province and were chosen randomly, to see where they were causing more damage and increasing its yield, these areas are: Tres Quebradas, Las Cruces, La Colorada, El Guasimo, Villa Lourdes, La Espigadilla, Los Angeles and Santa Ana.

As a high percentage of $100 \%$ of samples of weeds from different areas and varieties associated with the tomato production collected in the District of Los Santos taken as referent for this study; the most important were gather and grouped by taken into account the botanical families to which they belong, in the fallowing way: $17 \%$ Euphorbiaceae; 22\% Asteraceae and Fabaceae, (11\% each);8\% Poaceae; $18 \%$ Convolvulaceae, Malvaceae, Solanaceae (6\% each);20\% Amaranthaceous, Boraginaceous, Commelinaceae, Cucurbitaceous, Sterculiaceae (4\% each).

And the remaining $15 \%$ is homogeneously distributed among the families: Bignoniaceae, Capparidaceae, Cyperaceae, Loganiaceae, Onagraceae, Plantaginaceae, Portulacaceae, Rubiaceae Scrophulariaceae, Tiliaceae, Verbenaceae, Violaceae, Zygophyllaceae. Although the whole group of different varieties of collected and identified weeds could be used as a reservoir of biological entities, which should be considered in the design of strategies for farther researches to improve the agronomic management of the crop.

Keywords: weeds, identification, botanical families, species.
\end{abstract}

1 Instituto de Investigación Agropecuaria de Panamá. Centro de Investigación Agropecuaria de Azuero.

“Ingeniero Germán De León (CIAA). Ciudad de Panama, Panama.

2 Laboratorio de Investigación y Desarrollo, Empresas Melo, S.A. Ciudad de Panama, Panama.

* Autor por correspondencia: orlando.osorio@idiap.gob.pa; osorioboo11@gmail.com

Fecha de recepción: 19 junio, 2018.

Fecha de aceptación: 22 agosto, 2018.

DOI: http://dx.doi.org/10.4067/S0718-34292018005001401. Publicado en línea: 13-diciembre-2018. 


\section{Introducción}

La mayor producción de tomate industrial en Panamá se localiza en la provincia de Los Santos, en el distrito de Los Santos. En el Arco Seco, se siembra el $80 \%$ del total para la provincia (Departamento de Planificación, MIDA-2014). Esta región se dedica primordialmente a la producción de tomate industrial en la estación seca; en la cual se ha registrado una superficie sembrada de 147 hectáreas, con rendimiento promedio de 46.9 toneladas por hectárea (NestléPanamá, 2017). El costo de producción por hectárea es de alrededor de \$. 7,558.60, (Guerra y Aguilera, 2010) que incluye el $15 \%$ en el manejo de las arvenses mediante labranza o con la aspersión de pesticidas. Durante los meses de mayor precipitación se deja descansar el terreno para favorecer su recuperación y evitar las plagas fitopatológicas debidas al exceso de humedad.

La importancia de las arvenses en los sistemas de cultivos, tradicionalmente, ha sido desestimada. En el ciclo tradicional de cultivo de tomate, durante la estación lluviosa se favorece la germinación de las arvenses del banco de semillas del suelo y su crecimiento abundante. Antes del cultivo son eliminadas mecánicamente y con herbicidas. Durante la época de siembra, la adición de fertilizantes y la irrigación hacen que surjan nuevamente. La competencia con el cultivo por los recursos básicos del hábitat como son la luz solar, nutrientes, agua y espacio, aumento de los costos de producción, disminución del rendimiento, interferencia con las labores de cosecha, mala calidad de los frutos y reducen el valor de las tierras agrícolas (Salazar, 1990). Otro efecto, poco estudiado es su papel como hospederos de plagas y patógenos (Wisler and Norris, 2005). Sin embargo, se reconocen como refugio y alimento de insectos benéficos y de la vida silvestre, protegen el suelo de la radiación y la erosión en época de barbecho, reciclan minerales y nutrientes y mantienen la humedad (Altieri et al.,1979, Cock, 1996; Gliessman, 1998; Bernal, 2014), incluso algunas son fuente de alimentos y medicinas para el hombre. Por tanto, la identificación oportuna de las arvenses, asociadas a cultivos en áreas de clima cálido, es un factor fundamental para la determinación de umbrales económicos y biológicos, para el manejo y control de especies no deseadas en el cultivo y para su aprovechamiento (Montealegre, 2011). En este trabajo presentamos la diversidad de arvenses asociadas a los cultivos de tomate en el distrito de Los Santos, para contribuir con el desarrollo de estrategias de manejo integrado del cultivo.

\section{Materiales y métodos}

Área de colecta: El área de muestreo está ubicada en el Arco Seco, en el distrito de Los Santos en la Provincia de Los Santos, de la República de Panamá entre los 26 y 28 msnm. Las localidades muestreadas incluyen Tres Quebradas, Las Cruces, La Colorada,

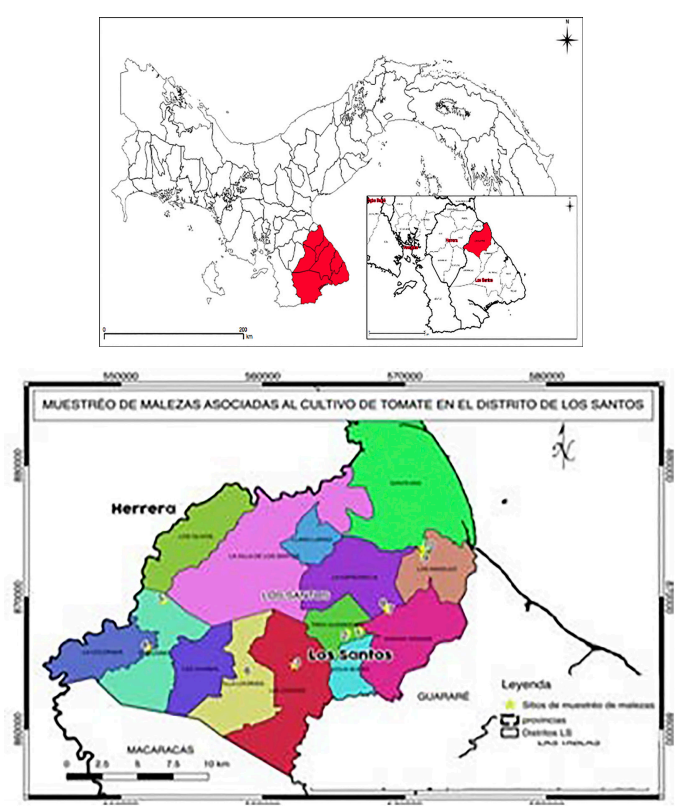

Figura 1. Ubicación de los sitios de muestreo en el Distrito de Los Santos, Provincia de Los Santos: 1.- Tres Quebradas 2.- Tres Quebradas 3.- La Limona 4.- La Colorada 5.-San Luis 6.-La Balita 7.- La Espigadilla 8.- La Lomita 9.- La Honda 10.- La Honda. 
Tabla 1.- Ubicación de las parcelas de tomate en el Distrito de Los Santos, Provincia de Los Santos.

\begin{tabular}{llccl}
\hline No. & Localidad (poblado, corregimiento) & \multicolumn{2}{c}{ Ubicación geográfica } & Altitud \\
\cline { 1 - 3 } & & $\mathbf{X}$ & $\mathbf{Y}$ & \\
\hline 1 & Tres Quebradas, Tres Quebradas & 566826 & 867510 & 64 \\
2 & Tres Quebradas, Tres Quebradas & 565965 & 867296 & 72 \\
3 & La Limona, Las Cruces & 562232 & 864848 & 83 \\
4 & La Colorada, La Colorada & 551897 & 866191 & 75 \\
5 & San Luis, San Luis & 553020 & 869712 & 46 \\
6 & La Balita, Villa Lourdes & 558716 & 864200 & 78 \\
7 & La Espigadilla & 568810 & 869035 & 47 \\
8 & La Lomita & 568687 & 869342 & 26 \\
9 & La Honda & 571284 & 873185 & 28 \\
10 & La Honda & 571283 & 873738 & 28 \\
\hline
\end{tabular}

El Guásimo, Villa Lourdes. La Espigadilla, Los Ángeles y Santa Ana. Se seleccionaron diez fincas dedicadas al cultivo de tomate industrial, seis fueron muestreadas en febrero y marzo de 2016 y cuatro en febrero y marzo de 2017 La ubicación de las parcelas se georreferenció usando un sistema americano de navegación y localización mediante satélite (Global Positioning System GPS) (Figura 1, Tabla 1).

Clima y topografía: La temperatura promedio de la región fue de $27.20^{\circ} \mathrm{C}$. Esta región se caracteriza por tener una baja precipitación pluvial anual que fluctúa entre 800 y $900 \mathrm{~mm}$, con una humedad relativa promedio de $82 \%$, radiación solar promedio de 198 $\mathrm{w} / \mathrm{m}^{2}$, presión atmosférica promedio de 1,009 bares $\mathrm{y}$ velocidad del viento promedio de $18 \mathrm{~m} / \mathrm{s}$ (Batista et al., 2017).

Colecta de plantas: Se colectaron en el interior $\mathrm{y}$ en el contorno de las parcelas de tomate (figura 2), un mínimo de tres plantas completas de cada especie, de preferencia con flores y frutos. En algunos casos se colectaron plantas adultas, que no contaban con estos órganos por no coincidir su ciclo reproductivo con el cultivo del tomate. Se colocaron en bolsas plásticas y se mantuvieron con hielo $\left(4^{\circ} \mathrm{C}\right)$ hasta llegar al laboratorio, donde se colocaron sobre papel periódico para su herbarización y fueron debidamente registradas.

Las características del cultivar de tomate T-8 son: alto potencial de rendimiento, entre 54.5 y 68 ton/ha, fruto de tipo perha grande y color rojo, ideal para la industria y consumo. fresco, y su alta tolerancia a la bacteria Ralstonia solanacearum (Smith), que produce la marchitez bacteriana. Su cultivo se recomienda en tierras bajas, fértiles, con poca pendiente, profundas y con facilidades de riego. Los descriptores fenotípicos de este cultivar son: hábito de crecimiento semideterminado; forma el fruto pera agrande; color del fruto inmaduro; verde claro; color de fruto maduro rojo; diámetro del fruto 8.0 - $8.6 \mathrm{~cm}$; días a floración 35 - 40 días después del trasplante(ddt); días a cosecha 60 - 75 ddt; rendimiento / planta 2.0 - $3.0 \mathrm{~kg}$; peso promedio / fruto 30 - $34 \mathrm{~g}$; números de frutos / planta 50 - 60; rendimiento / hectárea; 54.5 - 80 toneladas; duración de la cosecha 26 - 30 días (época seca); $\mathrm{pH}$ 5.0-6.0; brix; 6.0 - 7.0 (Guerra et al., 2013).
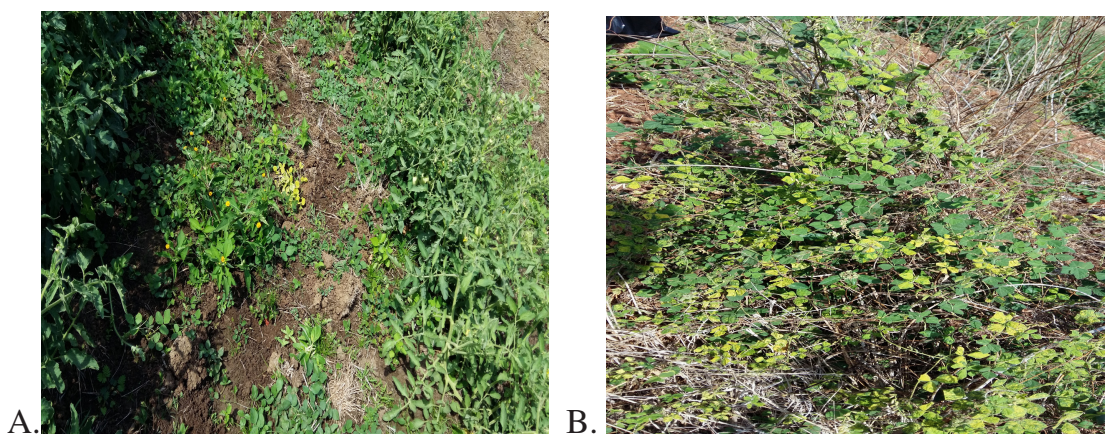

Figura 2. Arvenses en el cultivo. A.- Arvenses entre las hileras del cultivo.; B.- Arvenses en los bordes del cultivo. 
Identificación de arvenses: Todas las muestras colectadas, una vez codificadas fueron identificadas en el laboratorio del IDIAP y en el herbario de la Universidad Autónoma de Chiriquí (UNACHI). Se usaron claves taxonómicas para la identificación de las plantas siguiendo la metodología binaria latina expuesta por Linneo y especificadas en el Código Internacional de Nomenclatura Botánica.

\section{Resultados y discusión}

En las comunidades de arvenses muestreadas en las 10 parcelas de tomate variedad T8, se identificaron 22 familias de plantas dicotiledóneas (Tablas 2) y 3 familias de plantas monocotiledóneas (Tabla 3). En total 65 especies perteneciente a 25 familias (Tablas 2 y 3). La familia con mayor número de especies fueron

Tabla 2.- Arvenses dicotiledóneas asociadas al cultivo de tomate en el Distrito de Los Santos (2016-17).

\begin{tabular}{|c|c|c|}
\hline Familia & Nombre común & Nombre científico \\
\hline \multirow[t]{3}{*}{ Amaranthaceae } & Bledo espìnoso, bledo & Amaranthus spinosus $\mathrm{L}$. \\
\hline & Cadillo, caillo & Achyranthes indica (L)Mill. \\
\hline & Bledo & Amaranthus dubius Mart. \\
\hline \multirow{7}{*}{ Asteraceae } & Botón & Tridax procumbens $\mathrm{L}$. \\
\hline & Oreja ratón & Eleutheranthera rudelaris(Swartz). \\
\hline & Cervulaca, cirulaca, pariteña, & Baltimora recta $\mathrm{L}$. \\
\hline & Cervulaca, cirulaca & Melampodium divaricatum $(\mathrm{L})$. Rich \\
\hline & Cervulaquilla & Pectis elongata var. oerstedina (Rydb.) D.J. Keil \\
\hline & Flor amarilla & Chrysanthellum americanum var. integrifolium (Steetz) Alexander \\
\hline & Emilia, pincel & Emilia sonchifolia $(\mathrm{L}) \mathrm{DC}$ \\
\hline Bignoniaceae & Bejuco hormiguero & Batocydia unguis (L).Mart. \\
\hline \multirow[t]{2}{*}{ Boraginaceae } & Cola de alacrán & Heliotropium indicum $\mathrm{L}$. \\
\hline & Colita de alacrán & Heliotropium fruticosum $\mathrm{L}$. \\
\hline Capparidaceae & Quita ruina & Cleome viscosa $\mathrm{L}$. \\
\hline \multirow{4}{*}{ Convolvulaceae } & Flor lila & Jacquemontia evolvuloides Meisn. \\
\hline & Lilita & Evolvulus alsinoides (L.) L. \\
\hline & Trepadoya & Evolvulus convolvuloides (Willd. ex Schult.) Stearn. \\
\hline & Enredadero & Jacquemontia tamnifolia (L.)Griseb. \\
\hline \multirow[t]{2}{*}{ Cucurbitaceae } & Balsamina, balsamino & Momordica charantia $\mathrm{L}$. \\
\hline & Meloncillo & Cucumis melo L. \\
\hline \multirow{11}{*}{ Euphorbiaceae } & Ortiga macho & Jatropha gossypifolia $\mathrm{L}$. \\
\hline & Tamarindillo, tripa de pollo, flor escondida & Phyllanthus amarus Thonn. \\
\hline & Coquillo & Jathopha curcas $\mathrm{L}$. \\
\hline & Falsa cervulaca & Bernardia sidoides (Klotzsch) Müll. Arg. \\
\hline & Croton & Croton hirtus L' Hér. \\
\hline & Lechetrezna, piso & Chamaesyce thymifolia (L.) Millsp. \\
\hline & Flor de pascua & Euphorbia heterophylla $\mathrm{L}$. \\
\hline & Leche leche, lechecilla & Chamaesyce hirta $\mathrm{L}$. \\
\hline & Leche de sapo, lechita & Chamaesyce hypericifolia (L.) Millsp. \\
\hline & Hierba miona & Acalypha alopecuroides Jacq. \\
\hline & Caperonia & Caperonia palustris (L.) St. Hil. \\
\hline \multirow[t]{7}{*}{ Fabaceae } & Falsa dormidera & Aeschynomene americana $\mathrm{L}$. \\
\hline & Bejuquilla & Rhynchosia minima $(\mathrm{L}$.$) DC.$ \\
\hline & Oreja de ratón & Alysicarpus vaginalis (L.) DC. \\
\hline & Orejitilla & Desmodiun trifolium (L.) DC. \\
\hline & Bejuco, & Calopogonium muconoides Desv \\
\hline & Trebolito & Chamaescrista kunthiana (Schltdl. \& Cham.) H.S. Irwin \& Barneby \\
\hline & Pica - pica, itchweed & Mucuna pruriens (L)DC. \\
\hline Loganiaceae & Lombricera & Spigelia anthelmia $\mathrm{L}$. \\
\hline \multirow[t]{4}{*}{ Malvaceae } & Escobilla & Sida rhombifolia $\mathrm{L}$. \\
\hline & Malva & Malachra alceifolia Jacq. \\
\hline & Malvastrum & Malvastrum americanum $(\mathrm{L}$.$) Torr$ \\
\hline & Escobilla & Sida acuta Burmf. \\
\hline Onagraceae & Clavito de agua & Ludwigia octavalvis (Jacq.) P.H. Raven \\
\hline Plantaginaceae & Lechuguilla lila & Stemodia jorullensis Kunth. \\
\hline Portulacaceae & Verdolaga & Portulaca oleracea $\mathrm{L}$. \\
\hline Rubiaceae & Cansa peón & Spermacoce ovalifolia (M. Martens \& Galleotti) Hemsl. \\
\hline
\end{tabular}




\begin{tabular}{lll}
\multicolumn{2}{l}{ Continuación Tabla 2.} & \\
\hline Familia & Nombre común & Nombre científico \\
\hline Scrophulariaceae & Lechugilla amarilla & Mercadonia procumbens (Mill.) Small. \\
\hline \multirow{3}{*}{ Solanaceae } & Aji de monte & Solanum oxycarpum Schiede. \\
\cline { 2 - 3 } & Topetón, vejigón & Physalis angulata L. \\
\cline { 2 - 3 } & Aruña gato & Solanum siparunoide Ewan. \\
\cline { 2 - 3 } & Friega plato & Solanum torvum Swartz. \\
\hline Sterculiaceae & Escoba morada & Melochia pyramidata (L.) Britton. \\
\cline { 2 - 3 } & Malva lisa, hierba suave, hierba algodón & Waltheria indica L. \\
\hline Tiliaceae & $\begin{array}{l}\text { Escobidilla, escoba, té de monte, escobilla negra, yerba } \\
\text { de té }\end{array}$ & Corchorus orinocensis H.B.K. \\
\hline Verbenaceae & Cadillo, pega pega & Priva lappulaceae (L). Pers. \\
\hline Violaceae & Falsa espigelia,hierba del rosario, palmita de peña. & Hybanthus attenuatus (Humb. \& Bonpl. ex Willd.) Schulze-Menz. \\
\hline Zygophyllaceae & Falsa verdolaga, verdolaguita, false purslane & Kallstroemia maxima (L.) Hooker \& Arnott.
\end{tabular}

Tabla 3.- Arvenses monocotiledóneas asociadas al cultivo de tomate en el Distrito de Los Santos (2016-17).

\begin{tabular}{lll}
\hline Poaceae & Manisuri, tuquito & Rottboellia cochinchinensis (Lour.) Clayton \\
& Pasto bermuda & Cynodon dactylon (L.) Pers. \\
& Equinocloa & Echinochloa colonum (L.) Link. \\
& Plumilla. & Leptochloa filiformis $($ Lam.) Beauv. \\
& Paja blanca & Digitaria sanguinalis (L.) Scop. \\
\hline Commelinaceae & Piñita, siempre vive & Murdannia nudiflora (L). Brenan. \\
\hline Cyperaceae & Pimentilla, coquito & Cyperus rotundus L. \\
\hline
\end{tabular}

Euphorbiaceae (11); Fabaceae (7); Asteraceae (7), Poaceae (5), Malvaceae (4); Solanaceae (4); Convolvulaceae (4); Amaranthaceae (2); Boraginaceae (2); Cucurbitaceae (2); Sterculiaceae (2); las familias Tiliaceae, Loganiaceae, Bignoniaceae, Capparidaceae, Scrophulariaceae, Onagraceae, Plantaginaceae, Portulacaceae, Violaceae, Zygophyllaceae, Rubiaceae, Cyperaceae, Commelinaceae y Verbenaceae, cuentan con una especie (Figura 3). Algunas especies encontradas fueron: Portulaca oleracea L.; Euphorbia heterophylla L.; Heliotropium indicum L.; Physalis angulata L. (Fig. 4).

Del 100\% de las muestras de arvenses asociadas al cultivo de tomate, colectadas en el Distrito de Los Santos, el 17\% corresponde a la familias Euphorbiaceae; $22 \%$ Asteraceae y Fabaceae, (11\% c/u); 8\% Poaceae; 18 $\%$ Convolvulaceae, Malvaceae, Solanaceae (6\% c/u);
$20 \%$ Amaranthaceae, Boraginaceae, Commelinaceae, Cucurbitaceae, Sterculiaceae ( 4\% c/u); y el 15\% ( Fig. 6) restante se encuentra distribuido homogéneamente entre las familias: Bignoniaceae, Capparidaceae, Cyperaceae,Loganiaceae, Onagraceae,Plantaginaceae, Portulacaceae, Rubiaceae Scrophulariaceae, Tiliaceae, Verbenaceae, Violaceae, Zygophyllaceae. Las familias predominantes fueron las Euphorbiaceae, Asteraceae, Fabaceae y Poaceae.

Las áreas de cultivo de tomate en el Arco Seco de Azuero son manejadas de manera similar, habiendo una época de cultivo de tomate y otra en la cual se dedican al pastoreo, cultivo de otras hortalizas o se dejan en barbecho. Generalmente, el control químico o mecánico de las arvenses se realiza antes de iniciar la siembra, con la finalidad de evitar la competencia



Figura 3.- Número de especies por familia. 

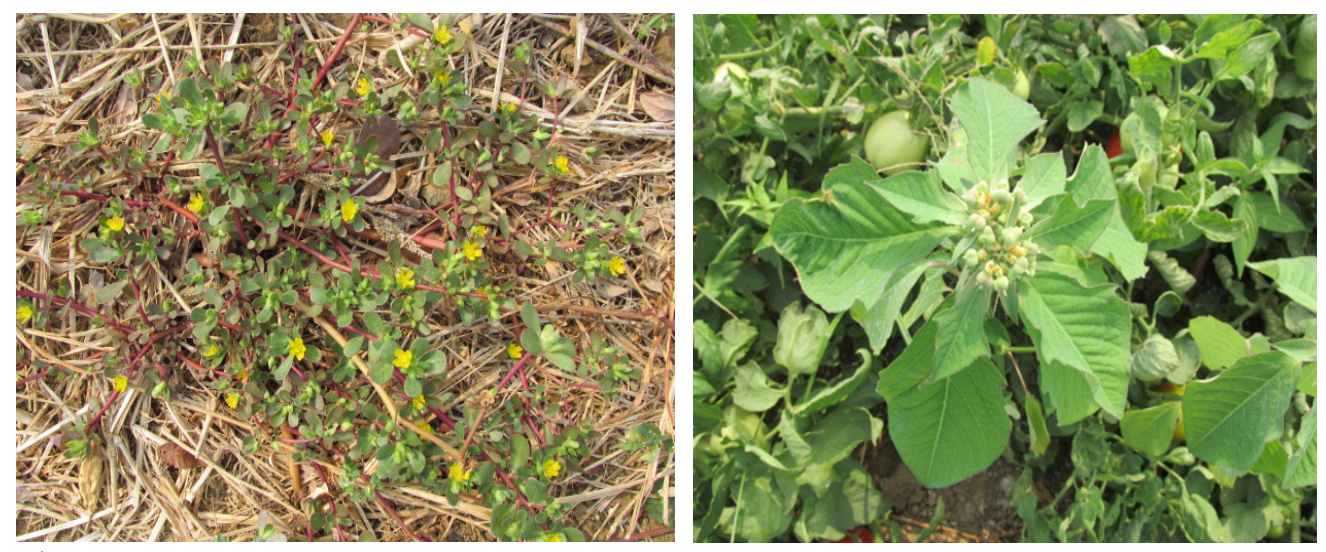

A.
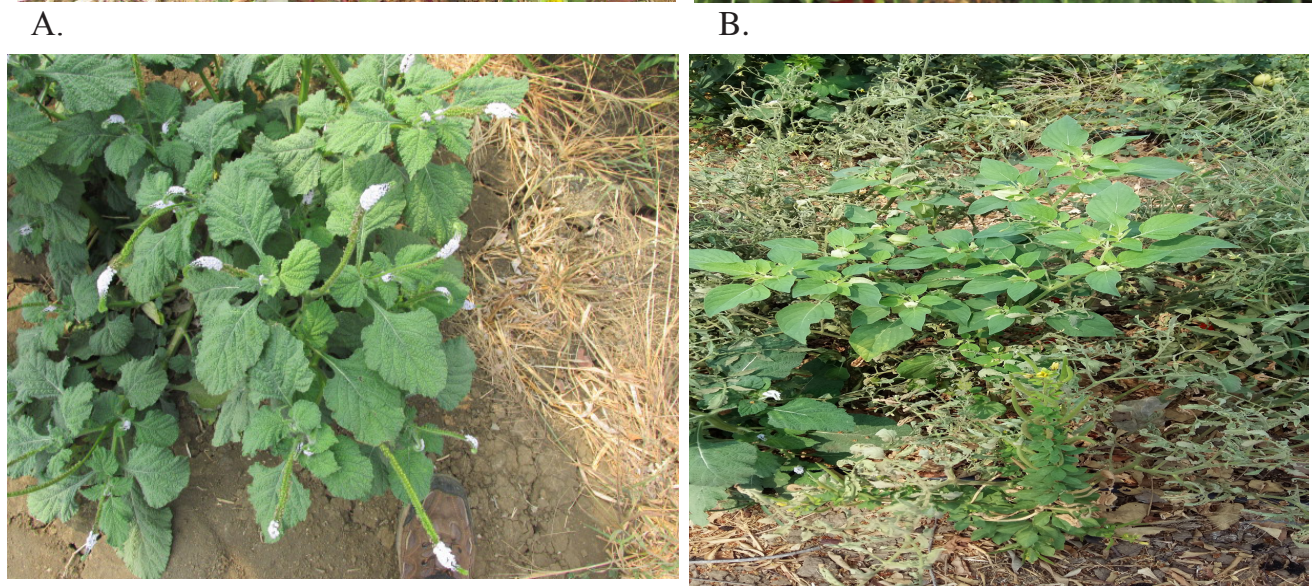

C.

D.

Figura 4.- Arvenses en el cultivo de tomate. A.- Portulaca oleracea L.; B.- Euphorbia heterophylla L.; C.- Heliotropium indicum L.; D.-. Physalis angulata L.

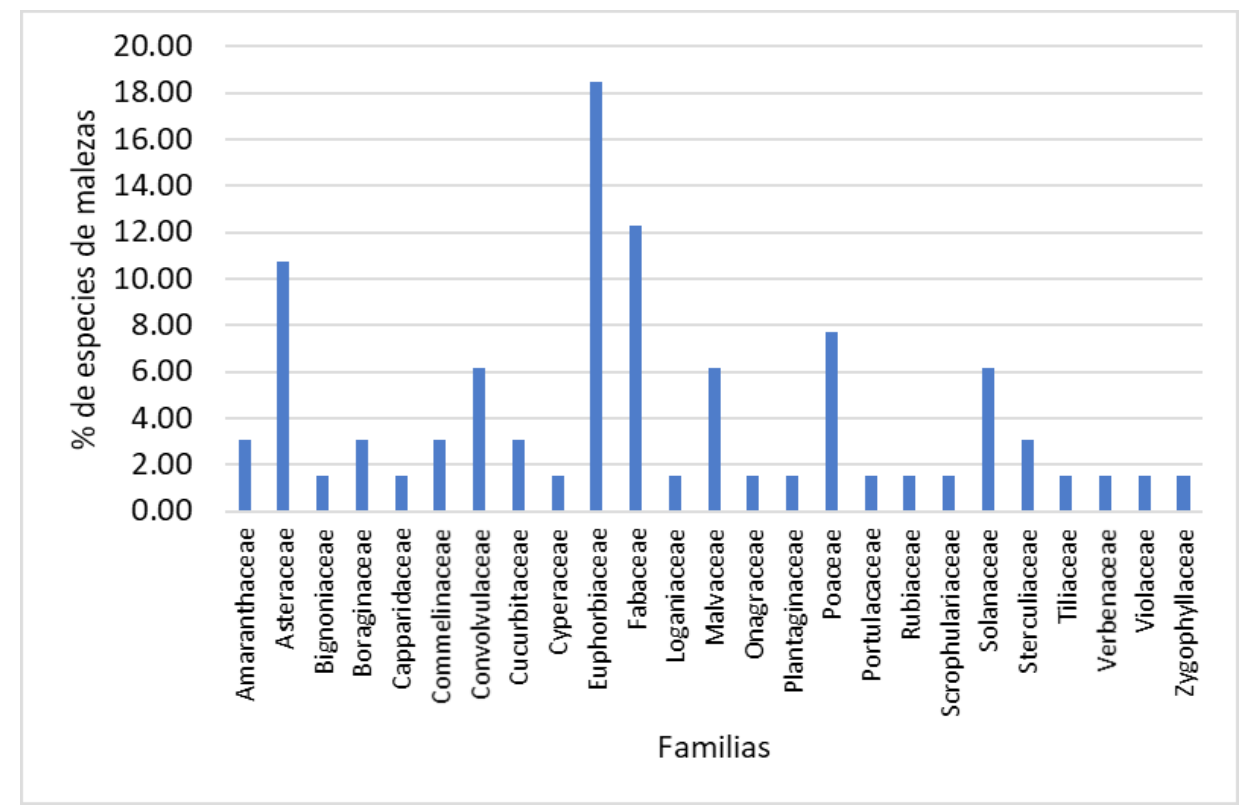

Figura 6. Porcentaje de especies por familia 
con el cultivo en las primeras etapas de desarrollo. Las parcelas muestreadas se encontraban en etapa de producción, por lo que las arvenses estaban en etapas vegetativas o de floración. Ante similares prácticas de manejo, características de suelo y clima, no se espera encontrar variaciones en la diversidad y abundancia de especies de una parcela a otra, como es evidente en los resultados obtenidos (Figuras 6).

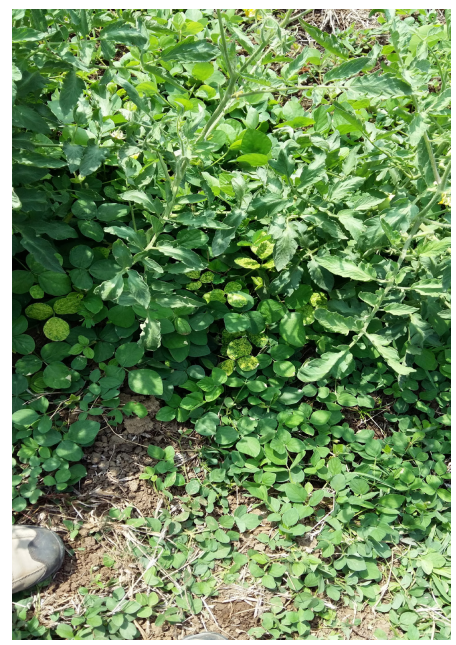

A.
Las arvenses son consideradas pestes o plagas, reservorios de patógenos o de vectores y su presencia está relacionada con la incidencia y distribución de las enfermedades de los cultivos (Wisler \& Norris, 2005). En las arvenses colectadas se observaron síntomas de infecciones virales, como mosaico amarillo (Figura 5). Siendo la infección por Begomoviruses una de las mayores limitantes en la producción del tomate en

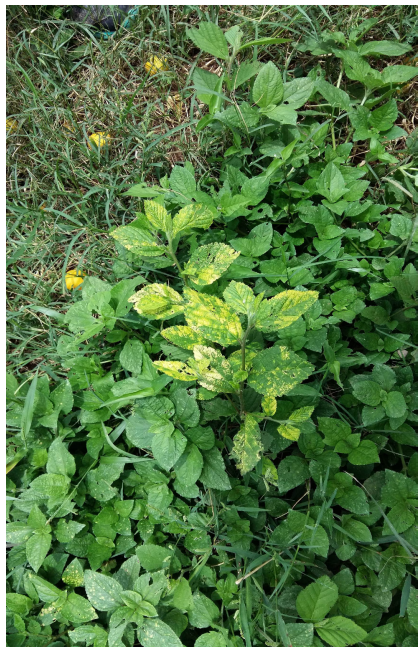

B.

Figura 5.- Arvenses dentro del cultivo de tomate que muestran síntomas de mosaico dorado. A. Rhynchosia minima (L.) DC.; B. Sida rhombifolia L.

el Arco Seco (Engel et al., 1998; Valderrama et al., 2002), fue notable la presencia de la mosca blanca en el cultivo y en algunas arvenses. Sería recomendable, hacer estudios del papel de cada una de las especies arvenses como reservorio de patógenos, sus vectores y enemigos naturales en los diferentes cultivos del Arco seco y evaluar las interacciones que se dan antes de eliminar completamente las arvenses del cultivo.

\section{Conclusiones}

En las comunidades de arvenses muestreadas en 10 parcelas de tomate variedad T8, ubicadas en el Arco Seco que corresponde al Distrito de Los Santos, se identificaron 25 familias de arvenses, 22 de plantas dicotiledóneas y 3 de plantas monocotiledóneas. En total, 65 especies, siendo las familias dicotiledóneas: Euphorbiaceae,Fabaceae,Asteraceaeylamonocotiledónea Poaceae, las de mayor número de especies.

Rhynchosia minima (L.) DC. es la especie de mayor distribución en las parcelas de tomate.
En las zonas donde no se establezca un manejo adecuado de las parcelas de producción, las malezas pueden incrementar exponencialmente su población.

Se deben hacer otras investigaciones que ayuden a conocer la biodiversidad de arvenses que existen en otras zonas agroecológicas del país y determinar los niveles críticos de cada especie y poder establecer estrategias de manejo de arvenses para minimizar el daño económico que ocasionan a los cultivos, enfocados en una agricultura sostenible a través del tiempo.

\section{Agradecimientos}

Este trabajo es parte del Proyecto ITE15-008 financiado por la Secretaría Nacional de Ciencia y Tecnología (SENACYT) de Panamá. Agradecemos el apoyo al Instituto de Investigación Agropecuaria de Panamá y al del Herbario de la UNACHI, a los profesores R. Rincón y R. Villarreal. 


\section{Literatura citada}

Altieri, M. A.; Whitcomb, W. H.

1979. The potential use of weeds in the manipulation of beneficial insects. HortScience, 14: 12-18.

Batista, A., Urriola D.; Batista, E.; Bustamante, S.; Díaz L.

2017. Red Agroclimática y Calidad de Aguas en Dos Sub Cuencas de Los Santos. Año 2015-2017. Programa: Investigación Innovación para la Competitividad del Agro Negocio (Azuero) Disponible: http://www.idiap.gob.pal. Consultado:23/nov/2016.

Bernal, J. A.

2014. Diversidad de especies de los Parasitoides de Bemisia tabaci (Genn.) (Hemíptera: Aleyrodidae) en cultivos y arvenses en Panamá. Tecnociencia, 16 (2): 9-31.

Cock, M. J.

1996. Control biológico de las malezas. En: Labrada, R.; Caseley, J. C.; Parker, C. (Eds.). Manejo de malezas para países en desarrollo. Estudio FAO. Producción y Protección Vegetal. Roma, Italia pp. 185-192.

Engel, M.; Fernandez, O., Jeske, H.; Frischmuth, T.

1998. Molecular characterization of a new whitefly-transmissible bipartite geminivirus infecting tomato in Panama. J. of General Virology, 79: 2313-2317.

Gliessman S.R.

1998. Agroecology: Ecological Processes in Sustainable Agriculture. Ann Arbor Press. Chelsea, MI, US.18p.
Guerra, J.A.; Aguilera V. 2010. Manual Técnico Manejo Integral del Tomate. Instituto de Investigación Agropecuaria de Panamá. 41p.

Guerra, J.A.; Him, P.; García, N; Castillo, A.; Batista, A.; Aguilera. 2013. Cultivar de tomate IDIAP T-8. Instituto de Investigación Agropecuaria de Panamá. Tríptico.

Ministerio De Desarrollo Agropecuario (MIDA).

2014. Informe departamento de planificación. 10 p.

Montealegre, F.A.

2011. Morfología de plántulas de arvenses de clima cálido. Ed. Produmedios. Bogotá D.C. Colombia.211 p.

NESTLÉ-PANAMÁ.

2017. Informe final Campaña de Tomate Industrial 2016-2017.15 p.

Salazar, L.C.

1990. Principales arvenses de arroz de secano en Panamá. Universidad de Panamá. 106p.

Valderrama, A.; Velásquez, A.; Fernández, O.

2002. Infección del Virus del rizado de las hojas del tomate (ToLCV-Pan) por Bemisia tabaci en Panamá. Manejo Integrado de Plagas y Agroecología (Costa Rica). 67: 67-71.

Wisler, G. C.; Norris, R. F.

2005. Interactions between weed and cultivated plants as related to management of plant pathogens. Weed Science, 56 (6): 914-917. 